\title{
Elemental Characterization of the Topmost Atomic Layer of Surface Using Doppler Broadening Spectroscopy
}

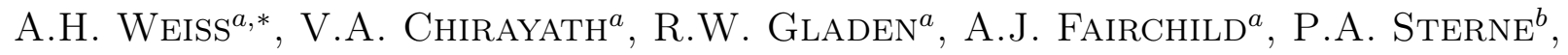 \\ S. Lotfimarangloo ${ }^{a}$, E. Perez ${ }^{a}$ And A.R. KOYmen ${ }^{a}$ \\ ${ }^{a}$ Department of Physics, University of Texas at Arlington, Texas-76019, USA \\ ${ }^{b}$ Lawrence Livermore National Laboratory, Livermore, California-94550, USA
}

This work is dedicated to Prof. Peter S. Pershan on his 85th birthday. His many pioneering contributions to physics research have provided an inspiring model.

\begin{abstract}
We present initial results that demonstrate the ability of Doppler broadening spectroscopy (DBS) to extract elemental information from the topmost atomic layer of a sample surface. Doppler broadening spectra from graphene grown on a polycrystalline $\mathrm{Cu}$ substrate was compared to the Doppler broadened spectra obtained after the partial removal of the bilayer graphene from the $\mathrm{Cu}$ surface. The Doppler broadening of the annihilation photopeak showed a clear difference between the as-inserted and the partially sputtered sample. We posit that this difference was due to the contribution of the gamma photons originating from the annihilation of positrons with the substrate $(\mathrm{Cu})$ electrons to the total Doppler broadened spectrum. The Doppler broadened spectrum of the partially sputtered sample was well fit with a line shape produced by combining the Doppler broadened gamma spectrum of the as-inserted sample with the theoretically calculated annihilation gamma photo peak from bulk $\mathrm{Cu}$. The fraction of the calculated Doppler broadened spectrum from bulk $\mathrm{Cu}$ in the fit spectrum is consistent with the increase in intensity of the $\mathrm{Cu} \mathrm{M}_{2,3} \mathrm{VV}$ Auger peak observed in the positron annihilation induced Auger electron spectra (PAES) of the partially sputtered sample. This suggests that analysis of the high momentum region of the gamma photopeak due to photons emitted following the annihilation of surface trapped positrons can provide a quantitative estimate of the elemental composition of the top surface. Our results provide evidence for the feasibility of using DBS as a new spectroscopic technique for the elemental characterization of the topmost atomic layer of surfaces.
\end{abstract}

DOI: 10.12693/APhysPolA.137.81

PACS/topics: 78.70.Bj, 71.60.+z, 41.75.Fr, 61.80.Fe, 82.80.Pv, 68.43.-h, 68.47.De

\section{Introduction}

The momentum of the annihilating electron-positron pair produces a deviation in the collinearity of the two annihilation gamma photons and Doppler shifts the annihilation photon energy as measured by the gamma detector [1]. The measurement of the angular correlation of two annihilation photons and the Doppler broadening of the annihilation gamma photopeak can therefore yield information about the momentum of the electronpositron pair. The momentum of the electron-positron pair is dominated by the momentum of the electron and therefore the analysis of the Doppler broadening provides chemical information of the site at which the positron annihilates [2]. Specifically, the high energy ("wing") region of the photopeak, which is strongly affected by contributions from core electrons, has been widely used to obtain chemical environment information of the defects or interfacial surfaces in the bulk or subsurface region of

\footnotetext{
*corresponding author; e-mail: weiss@uta.edu
}

the sample $[3,4]$. In this paper, we provide evidence of the feasibility of using Doppler broadening spectroscopy for chemical characterization of the topmost atomic layer of exposed surfaces.

Previous studies have shown that positron annihilation induced Auger spectroscopy (PAES) can be used to determine the elemental content of the topmost atomic layer of surfaces due to the trapping of positrons in an image potential-induced surface state before annihilation [5]. This property has recently been utilized to obtain the PAES data from a single layer of graphene on a $\mathrm{Cu}$ substrate [6]. The wavefunction of the surfacetrapped positrons decays rapidly and has appreciable overlap only with the electron wavefunctions spilling into the vacuum from the top surface. Weiss et al. [7] proposed that the gamma photon emitted following the annihilation of surface trapped positrons should provide highly surface selective chemical information and had shown that the high energy (equivalently high momentum) region of the annihilation gamma from multiple layers of a graphene sample on a Cu substrate was similar in shape to the high energy region of the theoretically calculated annihilation gamma from the bulk of graphite. Here, we extend the study further by comparing the 


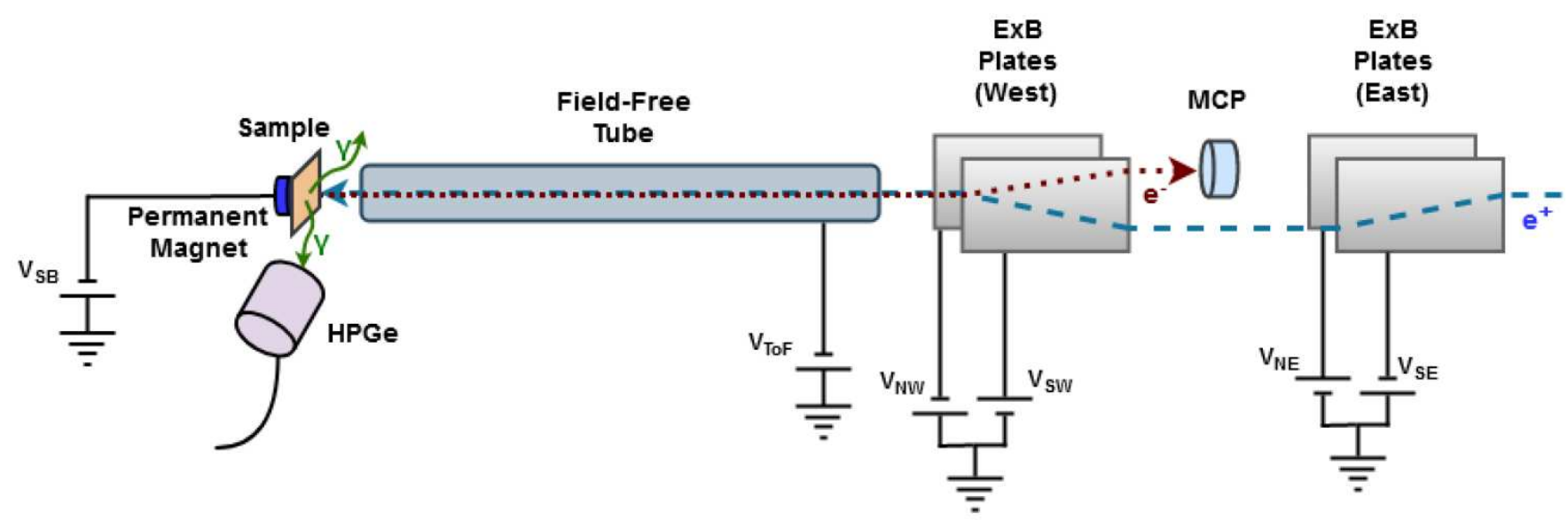

Fig. 1. Schematic of the sample side of the advanced positron beam equipped with the time of flight spectrometer. Monoenergetic low energy positrons are deflected around the microchannel plate (MCP) via $\boldsymbol{E} \times \boldsymbol{B}$ drift resulting from the combined action of an axial magnetic field $(\sim 0.005$ Tesla $)$ and transverse electric field generated by two sets of parallel plates. The positrons that exit the $\boldsymbol{E} \times \boldsymbol{B}$ system fly through a $\sim 3.0 \mathrm{~m}$ field-free tube before reaching the sample. The electrons ejected following the positron impact and/or annihilation traverse the field-free tube before getting bent up into the electron detector. The annihilation gamma is detected using a high purity germanium detector (HPGe). The time difference between the detected gamma signal and the electron detection signal from the MCP provides the measured flight time of the electron. The energy of the annihilation gamma ray was determined through an analysis of the amplitude of the HPGe pulse.

Doppler broadening spectra from bilayer graphene grown on a $\mathrm{Cu}$ substrate to that obtained from the same surface after partial exposure of the $\mathrm{Cu}$ substrate by sputtering in order to investigate the ability of Doppler broadening analysis to estimate the atomic composition of the top atomic layer of exposed external or internal surfaces.

\section{Experimental}

The advanced positron beam, including the data acquisition system used for the present experiment, has been described elsewhere [8,9] and only a brief description will be given here. A monoenergetic beam of low energy positrons are produced by a ${ }^{22} \mathrm{Na}$ source housed inside of a source holder with a parabolic face onto which a thin film of solidified Ne is grown [10]. The low energy positrons emitted from the Ne film are guided to the sample using an axial magnetic field generated by multiple solenoid coils. As shown in Fig. 1, positrons are drifted around the microchannel plate (MCP) electron detector by an $\boldsymbol{E} \times \boldsymbol{B}$ field generated using a transverse electric field and the axial magnetic field. The positrons pass through a field-free tube after exiting the $\boldsymbol{E} \times \boldsymbol{B}$ system and before reaching the sample. The energy of the incident positrons on the sample can be varied from $2.5 \mathrm{eV}$ to $20 \mathrm{keV}$ by biasing the sample (from $-0.5 \mathrm{~V}$ to $-20 \mathrm{kV}$ ) with respect to the field-free tube.

Electrons may be emitted from the sample surface following the transition of the positron to a surface (or bulk) state and/or after the Auger decay of annihilationinduced holes. The emitted electrons travel through the field-free tube before drifting up into the MCP by the action of the $\boldsymbol{E} \times \boldsymbol{B}$ system, producing a signal associated with the detection of the electron. The annihilation gamma photons are detected by the high purity germanium (HPGe) detector that produces a corresponding voltage pulse. The time difference between the signal from the HPGe detector and the MCP is used to produce a histogram of the measured flight times of the electrons, whereas the amplitude of the HPGe detector pulse is used to produce a histogram of the gamma energy. The time-of-flight spectra shown in the manuscript has been constructed using digital data acquisition and analysis techniques [8], whereas the gamma spectrum has been constructed using conventional analog electronics. The HPGe pulse used for the construction of the gamma energy histogram by analog electronics also served as the trigger for the digital data acquisition, making the two spectra representative of the same surface features. The HPGe detector, with $11 \%$ efficiency, has an energy resolution of $\sim 1.1 \mathrm{keV}$ at the $511 \mathrm{keV}$ photopeak and the timing spectrometer has a resolution of $\sim 22$ ns.

PAES and Doppler broadening spectra were collected from a bilayer graphene film grown on a polycrystalline $\mathrm{Cu}$ substrate purchased from ACS materials [11]. The sample was inserted into the vacuum chamber as received after which it was heated to between $375 \mathrm{~K}$ to $425 \mathrm{~K}$ in high-vacuum and then allowed to cool to room temperature before the data was collected. The measurements were repeated on the same sample after the partial removal of the graphene overlayer by sputtering with Argon ions. A visual inspection of the sample revealed that the sputtering did not completely expose the underlying $\mathrm{Cu}$ substrate over the full region irradiated by the positron beam due to a partial 
misalignment of the ion sputter gun. For the purpose of identification of the data in this paper, we will refer to the graphene on $\mathrm{Cu}$ sample before sputtering as "as-inserted" and to the same surface after sputtering as "partially sputtered." The positron measurements were carried out at a sample chamber pressure of less than $6 \times 10^{-8} \mathrm{~Pa}$.

\section{Results and Discussion}

Figure $2 \mathrm{a}$ and $\mathrm{b}$ show the PAES data collected from the sample surface before and after sputtering, respectively. The Auger spectrum from the sample before sputtering (Fig. 2a) was measured with a sample bias of $-0.5 \mathrm{~V}$ with respect to the field-free tube, and the maximum kinetic energy of the positrons that were incident of the sample was $\sim 7 \mathrm{eV}$. The PAES data from the partially sputtered sample was collected with $\sim 42 \mathrm{eV}$ positrons. We increased the kinetic energy of the positrons incident on the partially sputtered surface to improve the count rate and thus, to reduce the total data collection time maintaining the surface chemical composition of the partially sputtered surface during the experiment. In this setting, the field-free tube was also held at $-43 \mathrm{~V}$ relative to the sample which prevents electrons with energy less than $43 \mathrm{eV}$ from reaching the electron detector.

The Auger spectra from the sample before sputtering has three main peaks: the peak corresponding to the $\mathrm{M}_{2,3}$ VV Auger transition from $\mathrm{Cu}$, the $\mathrm{KVV}$ Auger peak from $\mathrm{C}$, and the KVV Auger peak from O. The presence of $\mathrm{Cu}$ in the as-inserted sample is attributed to small, but finite, overlap of the positron wavefunction with $\mathrm{Cu}$ atoms underneath the graphene overlayer [6]. After the partial sputtering of the bilayer graphene overlayer, all three Auger peaks remain. However, the intensity of the $\mathrm{Cu} \mathrm{M}_{2,3} \mathrm{VV}$ Auger peak measured relative to the intensity of the KVV Auger peaks from $\mathrm{C}$ and $\mathrm{O}$ increased by $\sim 15 \%$.

The Doppler broadening spectra collected from the asinserted and partially sputtered sample is shown in Fig. 3a along with the Doppler broadening spectrum representing the bulk of the $\mathrm{Cu}$ substrate. A background has been subtracted from all the gamma spectra following the prescription given by Mogensen et al. [12]. The "bulk"-like spectrum was collected by biasing the sample to $-20 \mathrm{kV}$ with respect to the field-free tube, whereas the Doppler broadening spectrum from the asinserted and partially sputtered sample surfaces was measured with the settings described above for PAES measurements. Particularly, the Doppler broadening spectra representing as-inserted and partially sputtered surfaces were collected simultaneously with the PAES spectrum, though not in true coincidence [8]. The voltage pulse from the HPGe detector used for the construction of gamma energy histogram using the analog electronics also served as the start signal for the time-offlight measurement using digital data acquisition techniques. The Doppler broadened annihilation gamma
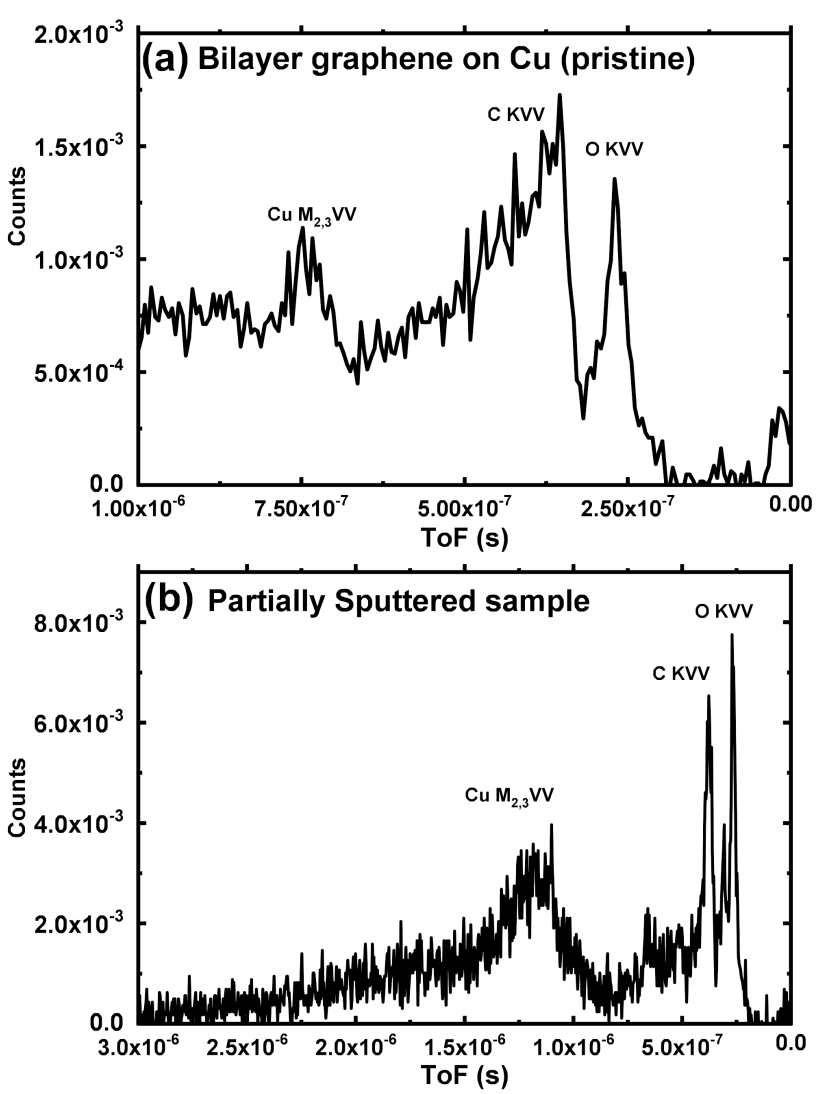

Fig. 2. (a) Positron annihilation induced Auger electron spectrum (PAES) measured from bilayer graphene grown on a $\mathrm{Cu}$ substrate. The spectrum shows clear peaks corresponding to the $\mathrm{C} \mathrm{KVV}$ and $\mathrm{O}$ KVV Auger peaks indicating the presence of carbon and oxygen in the topmost layer of the surface. There is also Auger peak corresponding to $\mathrm{M}_{2,3} \mathrm{VV}$ transition in $\mathrm{Cu}$ which we interpret as indicating some overlap of the wavefunction of surface trapped positrons with $\mathrm{Cu}$ atoms below a single layer of graphene. The maximum kinetic energy of the positron incident on the sample for this measurement was $\sim 7 \mathrm{eV}$ and the sample was biased to $-0.5 \mathrm{~V}$ with respect to the field-free tube. (b) PAES data measured after the partial sputtering of the graphene overlayer. The same three Auger peaks evident in the as-received sample remain after sputtering however, the intensity of the $\mathrm{Cu}$ MVV Auger peak may be seen to have increased significantly relative to the $\mathrm{C} \mathrm{KVV}$ and $\mathrm{O}$ KVV peaks. The measurement was performed with positrons that had a maximum kinetic energy of $42 \mathrm{eV}$. The field-free tube was held at $-43 \mathrm{~V}$ relative to the sample which prevents electrons with energy less than $43 \mathrm{eV}$ from reaching the electron detector.

spectra from the surface of the sample (bilayer graphene and partially sputtered surface) contains contributions from positrons annihilating in the surface state and from annihilations in the singlet or triplet Ps state in vacuum with little contribution from bulk of the sample. On the other hand, the Doppler broadening data collected with $-20 \mathrm{kV}$ on the sample predominantly contain bulk state annihilations with less than $3 \%$ of the total data collected resulting from surface state or bound 

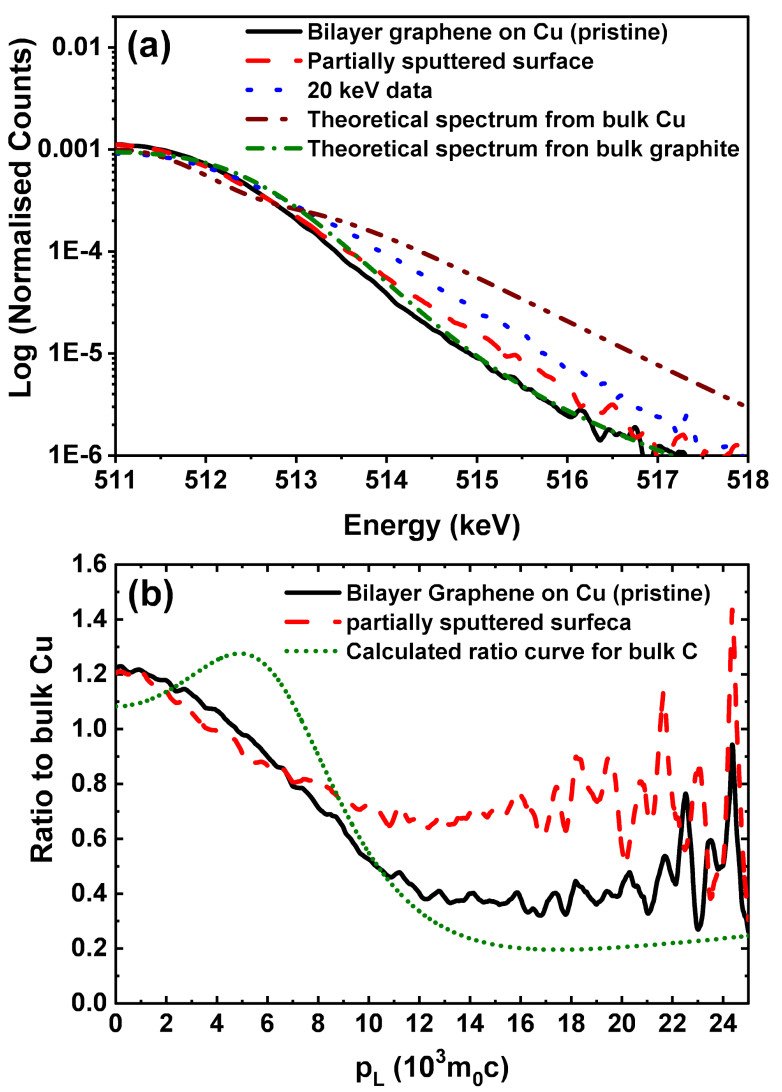

Fig. 3. (a) High energy region of the Doppler broadened annihilation gamma spectrum from different surfaces (bilayer graphene and partially sputtered surface) compared to the spectrum collected from the bulk of the substrate using positrons with $20 \mathrm{keV}$ energy and to the theoretically calculated spectrum from graphite and copper. The wing region of the spectrum from bilayer graphene matches with the wing region of the spectrum calculated from the bulk graphite. The Doppler broadened spectrum collected using $20 \mathrm{keV}$ positrons is markedly different from the bilayer graphene spectrum and the wing region of the spectrum is broadere and similar to the wing region of the theoretical Doppler spectrum from bulk Cu. (b) Ratio of the experimental Doppler broadening spectra from different surfaces taken with respect to spectrum collected with $20 \mathrm{keV}$ positrons. The experimental ratio curves have been compared to the ratio of the calculated Doppler spectrum from bulk graphite to that from bulk copper. The ratio curve of the bilayer graphene is comparable to the ratio curve of graphite in the high momentum region. The high momentum region of the partially sputtered surface clearly indicates that the spectrum has contribution from copper electrons at the surface.

Ps state annihilations. The experimental annihilation gamma photo peak has been compared to the theoretically calculated Doppler spectrum from bulk of graphite and $\mathrm{Cu}$ in Fig. 3a. Details of the calculation have been discussed in detail in previous publications [13]. The theoretical gamma spectra have been convoluted with a Gaussian representing the energy resolution of the detector at $511 \mathrm{keV}$.
The Doppler broadening spectrum from bilayer graphene is well described by the calculated spectrum from graphite, especially in the high momentum region. In contrast, the high momentum region or the "wing" of the Doppler broadened annihilation spectra from the partially sputtered surface and the spectra collected using $20 \mathrm{keV}$ positrons progressively broadens towards the wing region of the calculated spectrum from bulk $\mathrm{Cu}$. In order to amplify the features in each Doppler broadened spectrum a ratio curve analysis has been performed; the results of which are shown in Fig. 3b. The ratio of the experimental Doppler broadening spectra from different surfaces was taken with respect to the Doppler broadening annihilation spectrum measured using $20 \mathrm{keV}$ positrons. The calculated spectrum from bulk graphite has been divided by the calculated $\mathrm{Cu}$ spectrum to obtain the theoretical ratio curve. The ratio curve of both the as-inserted bilayer graphene and the partially sputtered surface is different from the theoretically calculated ratio at low momentum. However, at higher momentum ( $\left.>10 \times 10^{-3} m_{0} c\right)$, the shape of the asinserted surface is very similar to that of bulk graphite, showing the presence of graphitic carbon on the "as received" surface. The difference at the low momentum can be due to (i) the difference in bulk and surface projected valence electronic density and (ii) the fact that Doppler broadening spectrum at $20 \mathrm{keV}$ does not entirely represent the defect-free bulk $\mathrm{Cu}$ spectrum due to the presence of defects in the polycrystalline $\mathrm{Cu}$ substrate and small contribution from surface state/o-Ps pick off annihilations. The higher momentum region of the of the ratio curve from the partially sputtered surface shows a clear trend towards unity, signalling the presence of annihilations with $3 d$ electrons in $\mathrm{Cu}$; the absence of which in the graphitic sample is responsible for the dip seen at $\sim 15 \times 10^{-3} m_{0} c$ [14]. These results show that the high momentum region of the Doppler broadening spectrum reflects the surface chemical composition even when the spectra contain other features that affect the line shape in the low momentum region.

In order to determine the relative increase in annihilations with $\mathrm{Cu}$ atoms, the Doppler broadened annihilation spectrum from partially sputtered surface was fit using an empirical annihilation gamma line shape generated by combining the experimental Doppler spectrum from asinserted bilayer graphene sample with the Doppler spectrum calculated from bulk $\mathrm{Cu}$. The ratio of the empirical curve ( $85 \%$ the spectrum from the as-received sample and $15 \%$ the calculated $\mathrm{Cu}$ spectrum) taken with respect to the Doppler broadening annihilation spectrum measured using $20 \mathrm{keV}$ positrons is shown in Fig. 4 along with the ratio curve from the as-inserted surface and the partially sputtered surface. The fit captures most of the line shape of the partially sputtered surface especially the dip seen at $\sim 15 \times 10^{-3} m_{0} c$. The intensity of the calculated $\mathrm{Cu}$ spectrum in the fit curve is consistent with the increase in intensity of the $\mathrm{Cu} \mathrm{M}_{2,3} \mathrm{VV}$ Auger peak observed in the PAES spectrum from the partially 


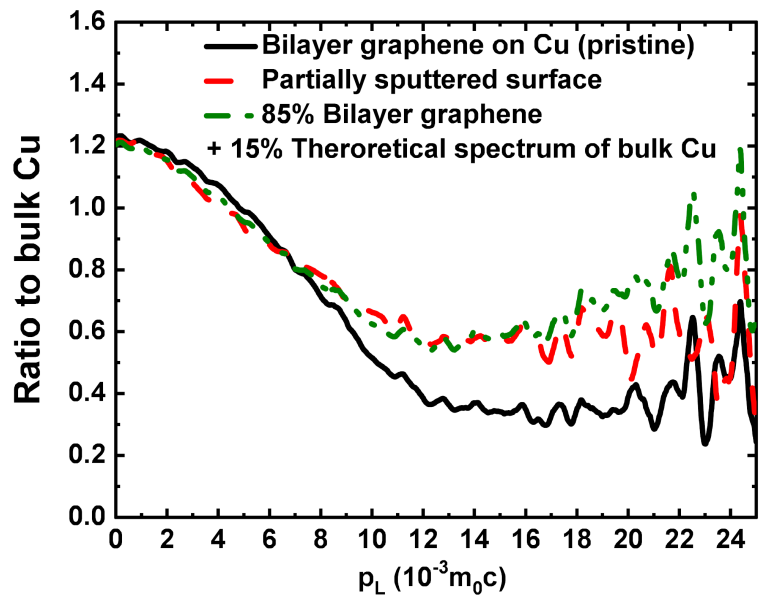

Fig. 4. Ratio of the Doppler broadening spectra from different surfaces taken with respect to spectrum collected at $20 \mathrm{keV}$. The experimental ratio curves corresponding to the partially sputtered surface have been reproduced by combining the experimental Doppler broadening spectrum from bilayer graphene with the calculated Doppler broadening spectrum from bulk $\mathrm{Cu}$. The amount of calculated $\mathrm{Cu}$ spectrum added is consistent with the increase in the intensity of $\mathrm{Cu}$ Auger peak obtained after the partial sputtering of the bilayer graphene overlayer. The good agreement between the measured Doppler broadening spectra with the model spectra consisting of a weighted sum of spectra from $\mathrm{C}$ and $\mathrm{Cu}$ result demonstrates the feasibility of using Doppler broadening spectroscopy to obtain an estimate of the relative elemental composition in the topmost atomic layer of a surface.

sputtered surface. These results demonstrate the ability of Doppler broadening spectroscopy to provide relative concentration of the elemental species at the topmost atomic layer of the surface.

Presently there is a need for a spectroscopic technique that can selectively address the surface modification on the inner surface of porous materials. The existing surface science techniques based on electrons, photons, or neutrons cannot provide direct surface selective information from the top layer of the inner surfaces of nano and micro porous materials. One of the reasons is that the escape depth of the excited electrons from which conventional surface analytic techniques derive their spectroscopic information is too shallow to investigate internal porous surfaces. Most other probes (photon or neutrons) provide information that is averaged over a range of depths and hence, is not surface selective enough when the pore ligaments are only a few nanometer thick. In contrast, positrons have a high affinity for trapping at these internal porous surfaces and the annihilation gamma rays can escape through thick samples and sample chambers carrying chemical information from the inner surface. We have shown above that the high momentum region of the annihilation gamma photopeak can provide a quantitative estimate of the elemental composition of the topmost atomic layer of external surface.
We posit that similar chemically sensitive information will be contained in the annihilation gamma photopeak even when they are escaping from the inner surfaces of porous materials. As a result, Doppler broadening spectroscopy can become a unique technique of characterizing the internal chemistry of the topmost atomic layer of these internal surfaces.

\section{Conclusion}

Doppler broadened annihilation gamma spectrum from bilayer graphene grown on a polycrystalline $\mathrm{Cu}$ substrate was compared to the Doppler broadened spectra obtained after the partial removal of graphene overlayer from the $\mathrm{Cu}$ surface. The Doppler broadened gamma spectrum from the partially sputtered surface shows a marked difference from the spectrum obtained from the bilayer graphene in the high momentum region due to annihilation with electrons belonging to the surface $\mathrm{Cu}$ atoms. Using an empirical fit to the gamma spectrum from the partially sputtered surface an estimate of the increase in $\mathrm{Cu}$ annihilations relative to the asinserted sample was obtained. The obtained estimate was found to be consistent with the increase in intensity of the $\mathrm{Cu} \mathrm{M}_{2,3} \mathrm{VV}$ Auger peak observed in the PAES data of the partially sputtered sample. Thus, an analysis of the gamma photopeak emitted following the annihilation of surface trapped positrons can provide a quantitative estimate of the elemental composition of the top surface. Based on these results, we propose that Doppler broadening spectroscopy can be used as an effective in-operando spectroscopic tool for the elemental characterization of the topmost atomic layer of the external surfaces of thin films and inner surfaces of microporous and nanoporous materials. Doppler broadening spectroscopy can fill in the existing lacuna in surface science for the characterization of the inner surfaces of porous materials.

\section{Acknowledgments}

This work was supported by Welch Foundation grant No. Y-1968-20180324. The initial research was funded by NSF grants DMR 1508719 and DMR 1338130.

\section{References}

[1] P.J. Schulz, K.G. Lynn, Rev. Mod. Phys. 60, 701 (1988).

[2] P. Asoka-Kumar, M. Alatalo, V.J. Ghosh, A.C. Kruseman, B. Nielsen, K.G. Lynn, Phys. Rev. Lett. 77, 2097 (1996).

[3] M. Maekawa, A. Kawasuso, J. Phys. Conf. Series 225, 012032 (2010).

[4] W. Shi, S.W.H. Eijt, C.S.S. Sandeep, L.D.A. Siebbeles, A.J. Houtepen, S. Kinge, E. Brück, B. Barbiellini, A. Bansil, Appl. Phys. Lett. 108, 081602 (2016).

[5] D. Mehl, A.R. Köymen, K.O. Jensen, F. Gotwald, A. Weiss, Phys. Rev. B 41, 799(R). 
[6] V.A. Chirayath, V. Callewaert, A.J. Fairchild, M.D. Chrysler, R.W. Gladen, A.D. Mcdonald, S.K. Imam, K. Shastry, A.R. Koymen, R. Saniz, B. Barbiellini, K. Rajeshwar, B. Partoens, A.H. Weiss, Nat. Commun. 8, 16116 (2017).

[7] A.H. Weiss, V.A. Chirayath, R.W. Gladen, A.J. Fairchild, M.D. Chrysler, P.A. Sterne, A.R. Koymen, AIP Conf. Proc. 2182, 050001 (2019).

[8] R. W. Gladen, V.A. Chirayath, A.J. Fairchild, A.R. Koymen, A.H. Weiss, Nucl. Instrum. Methods Phys. Res. A 162887 (2019).

[9] V.A. Chirayath, R.W. Gladen, A.D. McDonald, R.G. Greaves, A.J. Fairchild, P.V. Joglekar, S. Satyal, Z.H. Lim, M.D. Chrysler, T. Shead, B. Barnett, N. Byrnes, A.R. Koymen, A.H. Weiss, "A multi-stop time-of-flight spectrometer for the measurement of positron annihilation-induced electrons in coincidence with the Doppler broadened annihilation gamma" (manuscript under preparation).
[10] R.G. Greaves, C.M. Surko, Can. J. Phys. 74, 445 (1996).

[11] ACS Material, CVD Graphene on Copper Foil.

[12] O.E. Mogensen, T. Hirade, J. Phys. IV France 03, C4 (1993).

[13] P.A. Sterne, P. Asoka-Kumar, R.H. Howell, Appl. Surf. Sci. 194, 71 (2002).

[14] R.S. Brusa, W. Deng, G.P. Karwasz, A. Zecca, Nucl. Instrum. Meth. Phys. Res. B 194, 519 (2002). 